\title{
Efectos de los factores de riesgo sobre la interrupción de los estudios en jóvenes universitarios durante la covid-19
}

\section{Effects of risk factors on the interruption of studies in university students during COVID-19}

\author{
Víctor José Miranda Rodríguez ${ }^{1}$ \\ Universidad Tecnológica del Perú. Lima, Perú \\ c13208@utp.edu.pe \\ ORCID: https://orcid.org/0000-0003-0777-4351
}

Henry Hugo Alarcón Díaz ${ }^{2}$

Universidad Nacional de Educación Enrique Guzmán y Valle. Lima, Perú

halarcon@une.edu.pe

ORCID: https://orcid.org/0000-0003-1588-4390

Citar como: Miranda, V. y Alarcón, H. (2021). Efectos de los factores de riesgo sobre la interrupción de los estudios en jóvenes universitarios, durante la covid-19. Desde el Sur, 13(2), e0021

\section{RESUMEN}

La situación del estudiantado universitario, en la coyuntura actual de la pandemia, necesita desplegar esfuerzos para mantenerse como estudiantes y no seguir el destino que tuvieron los compañeros que abandonaron sus estudios. El objetivo de este artículo fue conocer los efectos de los factores de riesgo sobre la interrupción de los estudios en estudiantes universitarios durante la emergencia sanitaria por la covid-19. La unidad de análisis fueron estudiantes del cuarto ciclo de una universidad privada, y los resultados concluyentes arrojan que la deserción estudiantil se relaciona con cuatro factores: familiar, motivacional, institucional y económico. El factor institucional resultó influyente, al no ajustar su apoyo con el tema económico; sin embargo, el factor de riesgo económico fue el más relevante para que se tome la decisión de abandonar los

1 Diseñador gráfico periodístico, docente de la Facultad de Comunicaciones de la Universidad Tecnológica del Perú (UTP), magíster en Docencia Universitaria y Gestión Educativa por la Escuela de Posgrado de la UTP.

2 Licenciado en Educación, profesor principal de la Universidad Nacional de Educación Enrique Guzmán y Valle y doctor en Educación por la Universidad San Martín de Porres. 
estudios, ya sea de manera temporal o parcial, o también, como ocurre en muchas ocasiones, de manera definitiva.

\section{PALABRAS CLAVE}

Estudiante universitario, enseñanza superior, recursos económicos, pandemia

\section{ABSTRACT}

An issue faced by university students in the current situation resulting from the pandemic is how to continue as students, and not follow the fate of their classmates who have abandoned their studies. The goal of this article is to address the effects of risk factors on the interruption of studies in university students during the COVID-19 pandemic. The subjects of the study were students in their fourth semester at a private university, and the conclusive results indicate that student dropout rates are related to four factors: family, motivational, institutional and financial. The institutional factor was influential, given that support was not adjusted in response to financial issues; however, the financial risk factor was most relevant in the decision of students to abandon their studies, either temporarily or partially, and also, as occurred in several cases, permanently.

\section{KEYWORDS}

University students, higher education, financial resources, pandemic

\section{Introducción}

El presente estudio se enfoca en conocer los efectos de los factores que influyeron a determinar que un grupo de estudiantes de una universidad privada abandonen sus carreras por la crisis sanitaria que se vive en el Perú y el mundo. Según Esteche y Gerhard (2020), esta situación afectó no a cualquier sector social, cultural o económico, sino particularmente a los que estudian y trabajan, debido también a la falta de una reacción oportuna y rápida del sistema educativo peruano.

La educación en tiempos de pandemia significó un cambio en los sistemas educativos considerados como de autogestión de los aprendizajes, al dejar de lado las clases presenciales para pasar a la modalidad virtual. Este tránsito permitió el uso de aplicaciones y acciones tecnológicas ante la necesidad de continuar con el desarrollo de las clases. 
Esta obligada situación permitió utilizar diversas plataformas virtuales para la continuidad del aprendizaje. Los involucrados han tenido que desaprender para aprender nuevos formatos en la enseñanza superior, en una modalidad que en muchos casos es desconocida para los sujetos intervinientes en la educación (Unesco, 2020).

Las proyecciones sobre el aumento de estudiantes en el sector de la educación superior se vieron afectadas por la actual pandemia. Este hecho afectó y puso en riesgo la continuidad de los estudios en universitarios, pues en el transcurrir de los días muchos jóvenes dejaron de recibir clases de manera presencial, y tuvieron, al igual que los docentes, que aprender, sobre la marcha, el uso de estrategias de enseñanza y de aprendizaje, para llevar con relativo éxito sus asignaturas.

Por otro lado, la crisis económica que se desató en el país debido a la presencia del virus afectó a los sectores más vulnerables, lo cual se reflejó en menores ingresos para los padres de diversos estudiantes universitarios, que vieron reducidos sus salarios o, en el peor de los casos, perdieron sus empleos, y ya no contaron con los recursos para costear las pensiones de la universidad. A ello se sumó otro inconveniente: la incertidumbre académica, que significa tener dudas en la continuidad en gran parte de los elementos, las técnicas y las estructuras que rodean toda la educación y su proceso.

Este panorama obligó a recapitular lo relacionado con la capacitación en el uso de las nuevas tecnologías, tanto para docentes como para estudiantes, y hubo que adaptar los calendarios académicos y rediseñar las clases para ser desarrolladas en modalidad remota. La nueva normalidad había llegado y con ella se extendieron los horarios, se adaptaron los ciclos académicos y, en consecuencia, se reprogramaron las actividades diarias.

La deserción universitaria es un tema recurrente y viene desde tiempo atrás en diversos países. Ante esta problemática, las instituciones educativas iniciaron y siguen evaluando nuevos planteamientos pedagógicos para reducir sus porcentajes.

Existen diversas teorías acerca de la deserción. Según Tinto (1987), la decisión individual de permanencia en la universidad depende de un conjunto de variables determinadas por atributos de preingreso, como background académico en el colegio, variables socioeconómicas y culturales previas, y metas y compromisos de los estudiantes.

Los modelos sociológicos desarrollados por Bean (2001), Spady (1970) y Tinto (1975), a partir de dos conjuntos de factores, explican los motivos por los cuales los estudiantes deciden abandonar una institución universitaria. 
Otras investigaciones, como la de Montoya (1999), relacionan las condiciones socioeconómicas y el desempeño estudiantil con variables explicativas, y demuestran que los estudiantes de menor recurso económico son propensos a abandonar sus estudios académicos. La literatura sobre la deserción da cuenta de que existen numerosos trabajos de autores que analizan la deserción según su propia perspectiva, como se ilustra en la figura 1.

La deserción en la educación superior es un tema complejo de conceptualizar, pues aborda no solo una variedad de perspectivas, sino también una gama de diferentes tipos de abandono. Muchos autores la definen según sus objetivos y el problema a investigar (Tinto, 1989).

Loreto (2020) afirma que es posible reconocer un campo denominado educación y que resulta legítimo que existan diferentes saberes en ese ámbito, pero hay una disciplina propia llamada educación, cuyo objeto de estudio lo constituye el acto universal de enseñar, la pedagogía, que en sí entraña una complejidad por la cual confluyen diversos saberes, como la biología, la psicología, la sociología, la antropología, la filosofía, entre otros.

Una definición valiosa es la que plantea Himmel (2002), quien clasifica a la deserción como voluntaria (cuando el estudiante no informa a la institución que ha abandonado sus estudios) y como involuntaria (cuando

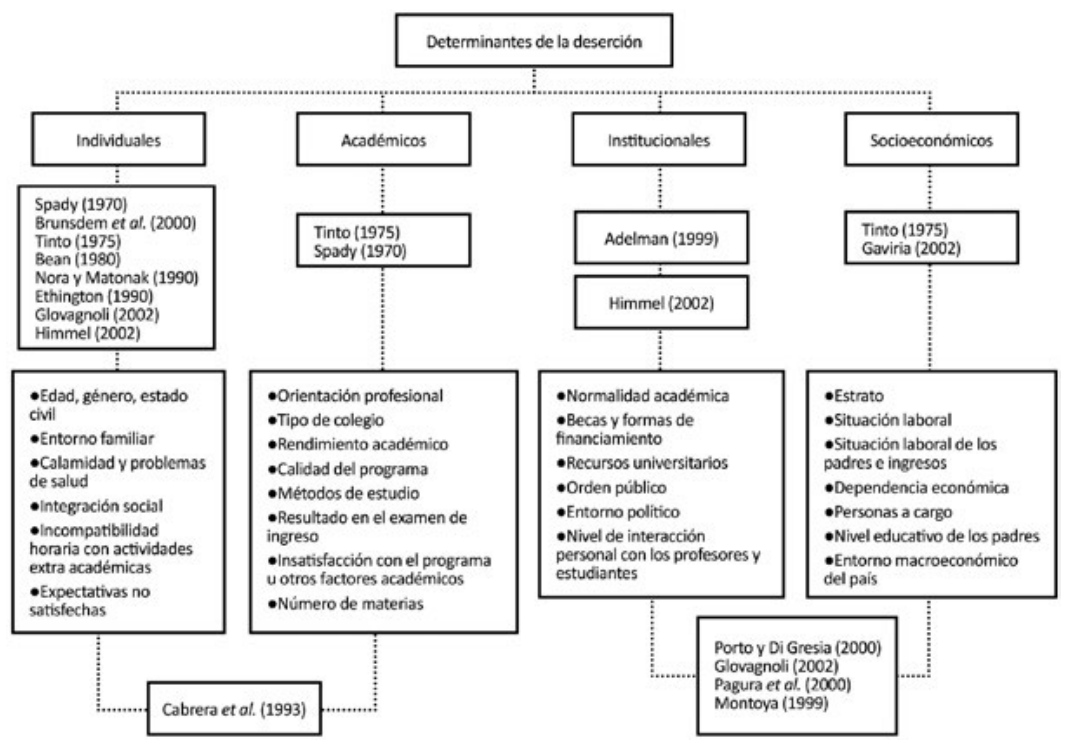

FIGURA 1. Estado del arte de los determinantes de la deserción estudiantil

Adaptado de Castaño, Gallón, Gómez y Vásquez (2008b) 
el estudiante debe abandonar la institución educativa por motivos reglamentarios). Entre ellos se considera el rendimiento académico.

También la deserción ha sido definida de acuerdo con el tiempo y el espacio. Respecto al tiempo, se clasifican en: a) deserción precoz, cuando el estudiante ingresa y no se matricula; b) deserción temprana, cuando el estudiante abandona sus estudios en los cuatro primeros ciclos de la carrera; y c) deserción tardía, cuando el estudiante abandona sus estudios del quinto ciclo en adelante (Castaño, Gallón, Gómez y Vásquez, 2004a). A continuación, ver la figura 2.

Según el espacio, la deserción se divide en: a) deserción interna o del programa académico, cuando el estudiante decide cambiar de carrera por otra dentro de la misma institución académica; b) deserción institucional, cuando el estudiante abandona la universidad; y c) deserción del sistema educativo (figura 3 ).

Cabe mencionar que el abandono de los estudios virtuales se vincula directamente con el factor económico por el costo/beneficio, ya que los estudiantes tienen en cuenta la retribución que podrían recibir a cambio

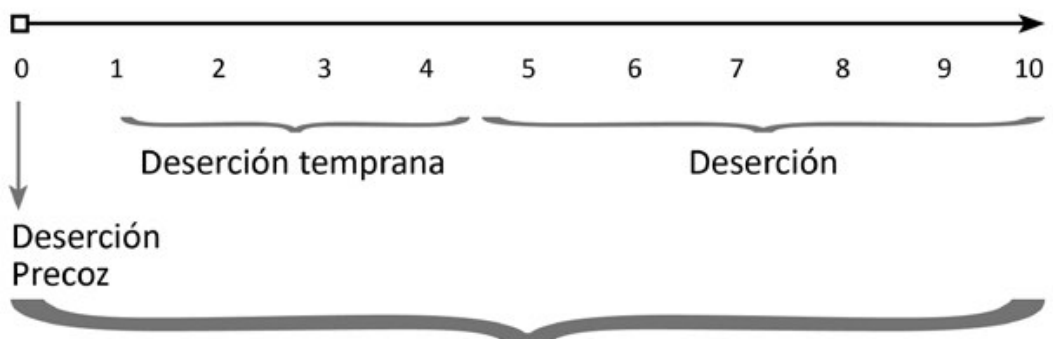

\section{Deserción total}

FIGURA 2. Clasificación de la deserción de acuerdo con el tiempo Fuente: Castaño, Gallón, Gómez y Vásquez (2004a)

Universidad № 2

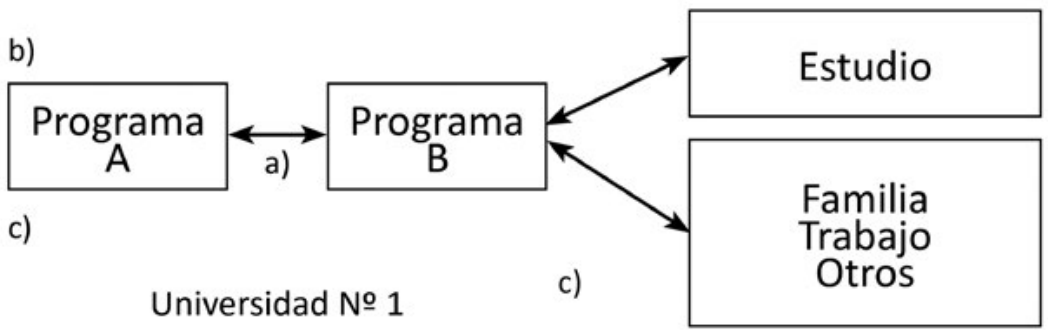

FIGURA 3. Clasificación de la deserción de acuerdo con el espacio 
del estudio, sea en lo social o en lo económico (Villareal, Castillo, Griffin y Rodríguez, 2011).

Es preciso incluir lo afirmado por Castro (2020), quien ofrece una interesante explicación sobre la importancia de la investigación. Por ejemplo, las ciencias de la salud no son las únicas que tienen materia pendiente por investigar, pues ahora, en tiempos de pandemia, las ciencias sociales y de la comunicación también tienen el imperativo de investigar el comportamiento de las personas, como, en este caso, la interrupción de los estudios en universitarios durante la pandemia por la covid- 19 .

A finales de diciembre de 2020, el Sistema Nacional de Evaluación, Acreditación y Certificación de la Calidad Educativa (Sineace) realizó un encuentro virtual sobre el impacto de la pandemia en las universidades. Según sus resultados, las cifras fueron poco alentadoras, ya que se comprobó que, hasta fines de setiembre de ese año, existían más de 174000 estudiantes en el país que habían interrumpido sus estudios universitarios. La cifra representa el $18,27 \%$ del total del universo de estudiantes universitarios, que son más de 955000 jóvenes. Ahora, si se compara esa cifra con la tasa de interrupción obtenida durante 2019 (12\%), el porcentaje o tasa de interrupción de 2020 es cerca de 6 puntos porcentuales mayor (Sineace, 2020).

Asimismo, según el informe del párrafo anterior, también se desprende que en las universidades públicas la tasa de deserción llegó a 9,85\%, en tanto que en las privadas el abandono alcanzó al 22,25\%, una cifra coincidente con Arellano. Entre las razones que el Ministerio de Educación (Minedu) atribuye para que los universitarios abandonen sus estudios están las económicas, familiares, vocacionales y académicas.

Ante el problema descrito anteriormente, la conectividad a Internet tiene, siempre, un rol importante. García (2020) señaló que las brechas al acceso del mundo digital incluyeron la dificultad para contar con tecnología, computadoras, teléfonos móviles con alta gama y, principalmente, con la conexión a Internet.

Otro de los factores a tener en cuenta en la interrupción o deserción es el carácter individual, que tiene que ver con la motivación. Para el estudiante, la parte afectiva puede ser vital en el logro de sus objetivos, al no encontrar alineación entre sus valores con los de la institución (Patiño y Cardona, 2012). Este efecto social tiene relación con la motivación de logro, con la meta de ser profesional y con las necesidades de los estudiantes referidas a cumplir sus aspiraciones o expectativas (Morán y Menezes, 2016). 
El estrés académico no está ajeno a esta dramática situación que se vive por la pandemia y, por ello, la reacción afecta en la motivación del estudiante. Este factor, asociado a la ansiedad, ha vulnerado la parte emotiva de los jóvenes respecto a su rendimiento académico. El virus ha provocado un impacto negativo en la salud mental de los estudiantes, docentes y todas las personas involucradas en el quehacer pedagógico.

McKay, Davis y Fanning (1998) definen al estrés como un «dolor emocional» producto de la «combinación de tres elementos: el ambiente, los pensamientos negativos y las respuestas físicas. Estos interactúan, de tal manera que hacen que una persona se sienta ansiosa, colérica o deprimida» (p. 9).

Los elementos descritos socavan la permanencia de los estudiantes en el sistema universitario, si no reciben el apoyo pertinente y oportuno por parte de las universidades y del ente rector, el Minedu.

Sobre el problema expuesto, el portal web del Minedu presenta una serie de recomendaciones para la continuidad del servicio educativo superior universitario. También las instituciones educativas a nivel superior promueven un freno a la deserción al reprogramar deudas y brindar becas de continuidad.

Montenegro (2020) afirma que el entorno donde se realizan los aprendizajes debe crear el contexto que facilite los aprendizajes, mediante el uso de recursos y material didáctico para afianzar los conocimientos.

Según la bibliografía investigada, la deserción se entrelaza con los factores de riesgo familiar, motivacionales, institucionales y económicos, aunque este último es el más influyente en la toma de decisión de los estudiantes para abandonar sus estudios, ya sea de modo temporal, parcial o definitivo, como ocurre en muchas ocasiones.

El factor de riesgo familiar se relaciona con la evaluación y clasificación de la vulnerabilidad de una familia. Coleman (1966) afirma que el background familiar (efectos del entorno familiar) se define a través de una serie de aspectos: el nivel socioeconómico familiar, la formación de los padres, los recursos culturales de que se dispone en el hogar y la estructura familiar.

El objetivo del presente estudio fue conocer los efectos de los factores de riesgo que determinaron que un grupo de estudiantes abandonaran sus estudios profesionales a causa de la covid- 19 .

\section{Materiales y métodos}

Las características del presente trabajo tienen un enfoque cuantitativo, que se inició con el problema de estudio. Luego se precisó su objetivo, 
se enriqueció con el marco teórico y se recolectaron datos, que, a la luz de los resultados del cálculo de valores o magnitudes encontrados, se contrastó con los aportes teóricos.

El trabajo tiene un carácter o alcance descriptivo, puesto que describe y manifiesta las características del objeto de estudio analizado, con base en la información recogida (Hernández, Fernández y Baptista, 2014).

La población fueron alumnos de la carrera de Ciencias de la Comunicación de la Universidad Tecnológica del Perú (UTP) a finales de 2020. La muestra estuvo conformada por 50 estudiantes, elegida por conveniencia y que «permite seleccionar aquellos casos accesibles que acepten ser incluidos. Esto, fundamentado en la conveniente accesibilidad y proximidad de los sujetos para el investigador»(Otzen y Manterola, 2017, p. 230).

Para recoger los datos, en virtud de la intención o alcance de la presente investigación (Diaz, Suárez y Flores, 2016), se aplicó un cuestionario tipo Likert y de múltiples opciones (para el resto de las preguntas), que fue subido a la plataforma de Google con la herramienta de formularios, con el propósito de obtener la información requerida. Una vez obtenidos los resultados de los estudiantes universitarios, se procedió a colocarlos en Google Data Studio (formulario con formato de hoja de cálculo), que permitió de forma sencilla crear gráficos a los resultados de las respuestas obtenidas.

En cuanto al protocolo de recojo de datos, se tuvo cuidado en los enunciados de las preguntas, y se validó previamente el cuestionario en cuanto al constructo, criterio y contenido. Asimismo, se tomaron fuentes de información recogidas directamente para la presente investigación, así como las fuentes de datos secundarios, basadas en fuentes documentales concernientes al tema de interés.

\section{Resultados}

Tras analizar los resultados expresados en las figuras respectivas, se afirma que los factores de riesgo que más influyeron en los estudiantes, en la determinación de abandonar sus estudios superiores durante la pandemia, fueron el económico, el motivacional y el institucional.

En tal sentido, se desprende que el factor económico, con el $40,7 \%$, es el más influyente para decidir desertar. Le siguen el motivacional $(33,3 \%)$ y el institucional $(20,4 \%)$. El factor familiar no intervino en el abandono de los estudios durante 2020 (figura 4).

Respecto al factor motivacional, el estudiante siente que el modo de enseñanza virtual no tiene el mismo efecto positivo o favorable que el modo presencial, pues necesita mayor acercamiento hacia el docente, y 
que la calidad de enseñanza, al no ser la adecuada, podría influenciar en su determinación de dejar sus estudios (figura 5).

El factor institucional está en actitud vigilante por parte del estudiantado y de los padres de familia. De no haber un descuento en el precio de las pensiones, podría considerarse como una dificultad para regresar a clases en los siguientes semestres. De otro lado, si la entidad educativa no establece nuevas estrategias metodológicas para la enseñanza, no brinda facilidades de pago ni facilita la accesibilidad virtual al estudiantado, la retención de los universitarios fracasará (figura 6).

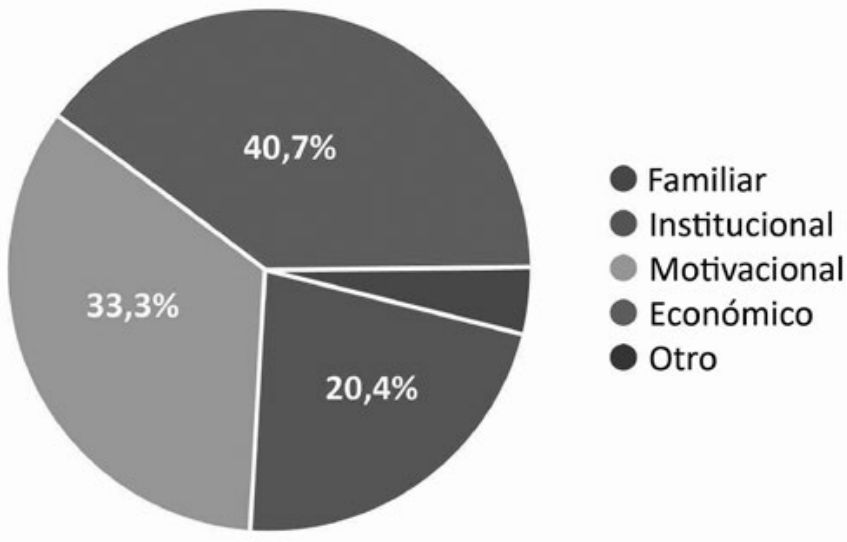

FIGURA 4. Distribución de la data. Aspectos que podrían determinar la continuidad de sus estudios Fuente: elaboración propia

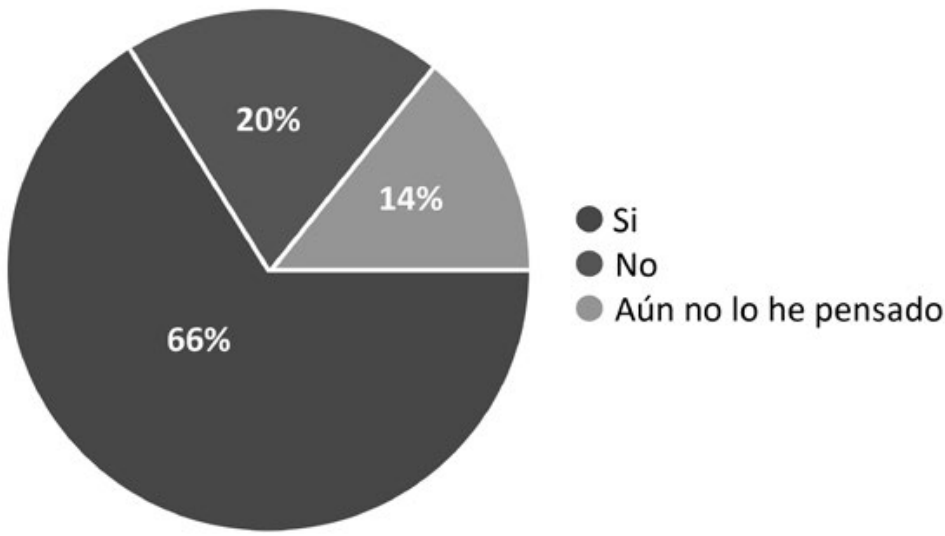

FIGURA 5. Distribución de la data. Iniciado el ciclo académico, ¿considera que la carrera que estudia se ajusta a sus intereses motivacionales?

Fuente: elaboración propia 


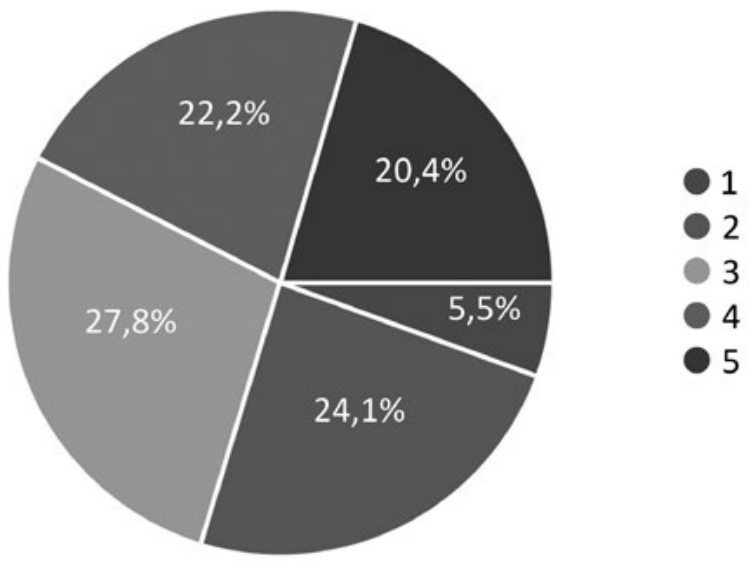

FIGURA 6. Distribución de la data. Sobre la calidad de servicio. Nivel de satisfacción con la institución educativa (5 es el máximo)

Fuente: elaboración propia

Muy influyente $\square$ Influyente $\square$ Moderadamente influyente $\square$ Poco influyente $\square$ Nada influyente
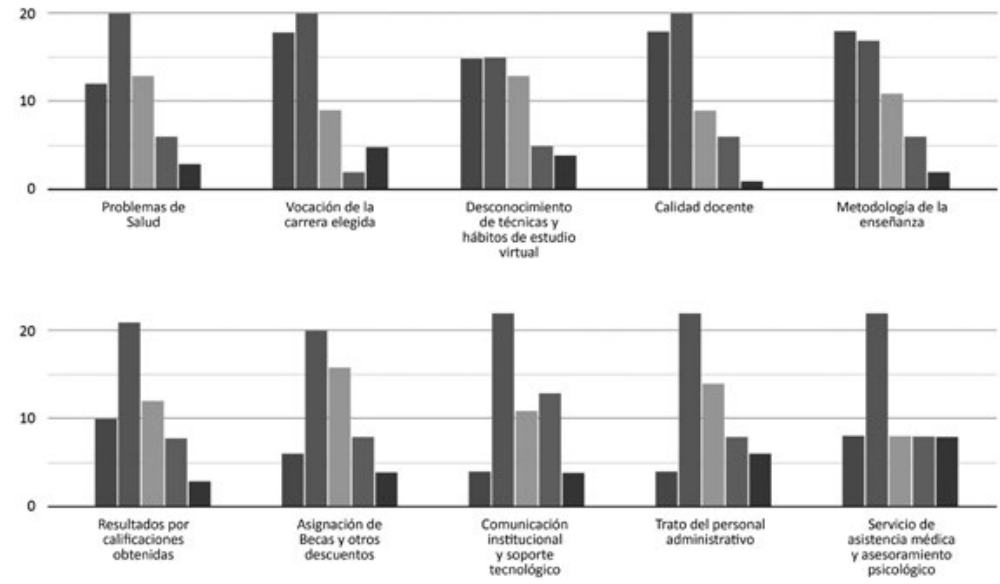

FIGURA 7. Distribución de la data. Aspectos que podrían determinar la continuidad de sus estudios Fuente: elaboración propia

La información analizada también indica que el factor familiar no es motivo de una posible deserción. Existen factores vinculados a lo económico, motivacional e institucional que se agregan a los de la interconectividad, como el pago de Internet, el ancho de banda que dificulta el acceso a la plataforma virtual, el tiempo de conexión y los escasos conocimientos 
y recursos tecnológicos por parte del estudiante, como contar con una computadora o laptop con disco duro, que soporten la instalación de programas actualizados necesarios para sus asignaturas.

\section{Discusión}

Desde la declaración de la pandemia el 11 de marzo de 2020 por la Organización Mundial de la Salud (OMS), la presencia de este nuevo virus afectó a todos los sectores, especialmente a los relacionados con la salud, la economía y la educación.

El sorpresivo cambio de las clases presenciales a clases a distancia o virtuales tomó desprevenidas a muchas instituciones educativas, a docentes, estudiantes y a los mismos padres de familia, y colocó a todos en un escenario inimaginable. Las medidas de prevención se realizaron de manera extrema. Ninguna persona deseaba contagiarse y mucho menos por acudir a clases. Las instituciones educativas cerraron sus instalaciones y se plantearon nuevas formas de educar, porque la educación no podía detenerse.

El objetivo trazado al inicio, de conocer los efectos de los factores de riesgo sobre la interrupción, se ha respondido e ilustrado en las figuras respectivas.

La educación virtual es considerada como una modalidad educativa que optimiza y mejora la calidad de la enseñanza y del aprendizaje, puesto que toma en cuenta la flexibilidad, es decir, los estudiantes pueden organizar sus tiempos y espacios de estudio en función de su disponibilidad (Lara, 2002). Al respecto, según la figura 5, que tiene que ver con el factor motivacional, los estudiantes no encuentran motivación en la modalidad virtual y apuestan más por la presencial. Aquí podemos encontrar algunas respuestas; por ejemplo, los estudiantes reciben, en gran parte, una enseñanza remota y no virtual. Además, existen estudiantes que no cuentan con los equipos adecuados, o la institución y los docentes no están implementados en un $100 \%$.

Con lo expuesto en el párrafo anterior, es importante también destacar el desempeño que toman los estudiantes para que la educación remota sea un éxito. Deben estar predispuestos, motivados y sobre todo ser responsables, a fin de organizar y gestionar sus tiempos para desarrollar sus actividades académicas oportunamente. Existen muchos factores para lograr ese objetivo; sin embargo, lo más importante es la actitud que ponga el estudiante en cada acción (Hernández, Fernández y Pulido, 2018).

En lo concerniente a los factores económicos, motivacionales e institucionales, sumados a la interconectividad, se expresan en la figura 7. El vecino país de Chile tenía mayor penetración digital, pero la desigualdad 
se ha evidenciado a través de las diferencias de cobertura, calidad y dispositivos (desconexión y brecha digital en Chile durante la epidemia de la covid-19). En nuestro país, para contrarrestar este problema, algunas universidades han entregado dispositivos electrónicos y chips, que mejoran la conexión de sus estudiantes con menos recursos. Otras instituciones han brindado becas o beneficios de conectividad, así como licencias y software para la docencia virtual, reforzamiento de las TIC, etc.

A nivel nacional, Ramírez (2009, como se citó en Liu, 2018), sostuvo que la causa principal para la deserción universitaria en la Universidad Nacional Mayor de San Marcos (UNMSM) era el problema económico (44\%), seguida por la falta de vocación profesional a la carrera (31\%). El factor institucional está en tercer lugar con las expectativas defraudadas en la formación (15\%) y el bajo rendimiento académico (8\%). En tal sentido, existe una coincidencia de la orientación de los resultados con el presente estudio, que se desprende de la figura 4, referido a que el factor económico, con el $40 \%$, es el más influyente para decidir desertar. Le siguen el motivacional (34\%) y el institucional (22\%). El factor familiar no influye en el abandono de los estudios durante 2020.

Al respecto, una de las conclusiones de la investigación realizada por Uribe (2021) afirma que hubo un incremento importante durante el periodo académico 2020-I, sobre la interrupción de estudios de los estudiantes universitarios, especialmente de aquellos que provienen de instituciones de gestión privada y en donde el grupo etario determinante fluctúa entre 19 y 23 años.

Hay que resaltar que las universidades, desde el punto de vista de los estudiantes, no han otorgado mayores beneficios económicos debido a la coyuntura. En las instituciones particulares no se han concretado descuentos en las pensiones, y en las públicas solo se ha exonerado un escaso porcentaje en el pago de la matrícula. Este hecho se encadena con los resultados expresados en la figura 6 , que vincula al factor institucional con la crisis económica y laboral, porque no se evidencian descuentos significativos en el precio de las pensiones. Este caso podría considerarse como un inconveniente para que los estudiantes se matriculen en los siguientes semestres académicos. Si la institución educativa no establece nuevas estrategias para la enseñanza y el aprendizaje, no brinda facilidades de pago, ni tampoco facilita la accesibilidad virtual al estudiantado, la permanencia de los estudiantes estaría en alto riesgo.

La consultora Arellano \& Marketing realizó un sondeo a estudiantes universitarios y de institutos en marzo y abril de 2020, para conocer qué decisión tomarían frente a la crisis económica ocasionada por la covid-19. El resultado arrojó que los estudiantes continuarán sus estudios pasada 
la cuarentena, pero de manera presencial, y que el $22 \%$ postergarían sus estudios. Entre los estudiantes que iniciarán sus estudios superiores, un $47 \%$ dijo que los pospondrían.

Cabe mencionar que el presente estudio es de carácter descriptivo. Se pueden también generar otros estudios de carácter correlacional, que involucren otras variables como el desarrollo de competencias, las estrategias de enseñanza, aprendizaje y de acompañamiento académico, el estrés académico, la salud mental, la calidad educativa, la autoeficacia, etc.

A manera de sugerencia, ante la crisis sanitaria, las universidades consideran, en un futuro cercano, un nuevo modelo educativo pospandemia, donde se involucrarán las fases de enseñanza presencial y remota. A lo expuesto, se debería replantear la implementación de estrategias de enseñanza y de aprendizaje pertinentes, centradas en el estudiante. En tal sentido, será necesario rediseñar estrategias, capacitar a los docentes y a las instituciones, además de brindar un soporte tecnológico de fácil acceso a los estudiantes, para así influenciar en su retención.

\section{Contribución de autoría}

Víctor José Miranda Rodríguez es el autor principal del artículo y responsable de la estructura y redacción del documento. Henry Hugo Alarcón Díaz participó como coautor del artículo.

\section{Fuente de financiamiento}

Autofinanciado.

\section{Potenciales conflictos de interés}

Ninguno. 


\section{REFERENCIAS BIBLIOGRÁFICAS}

Bean, J. y Eaton, S. B. (2001). The psychology underlying successful retention practices. Journal of College Student Retention Research, Theory \& Practice, 3(1), 73-89.

Castaño, E., Gallón, S., Gómez, K. y Vásquez, J. (2004a). Deserción estudiantil universitaria: una aplicación de modelos de duración. Lecturas de Economía, (60), 39-65. https://www.redalyc.org/articulo.oa?id=155217798002

Castaño, E., Gallón, S., Gómez, K. y Vásquez, J. (2008b). Análisis de los factores asociados a la deserción estudiantil en la Educación Superior: un estudio de caso. Revista de Educación, 345, 255-280. https://www.researchgate. net/publication/28200111_Analisis_de_los_factores_asociados_a_la_desercion_estudiantil_en_la_Educacion_Superior_un_estudio_de_caso

Castro, R. (2020). COVID-19. Narrativas y consideraciones sociales, culturales y políticas. Los otros virales. Desde el Sur, 12(1), 9-10. https://revistas. cientifica.edu.pe/index.php/desdeelsur/article/view/641

Diaz, C., Suárez, G. y Flores, E. (2016). Guía de investigación en educación. Vicerrectorado de Investigación de la Pontificia Universidad Católica del Perú. http://repositorio.pucp.edu.pe/index/handle/123456789/133219

Esteche, E. y Gerhard Y. (2020). Factores que inciden en la educación virtual en tiempos de pandemia (COVID-19) de los estudiantes universitarios de una universidad privada. Revista Iberoamericana de Docentes, 1-15. http://formacionib.org/faactoresqueinciden.pdf

García, F. (2020). El sistema universitario ante la COVID-19: Corto, medio y largo plazo. Universidad Blog de Studia XXI. https://bit.ly/2YPUeXU

Hernández, R., Fernández, C. y Baptista, M. (2014). Metodología de la investigación. (6. a edición). McGraw-Hill e Interamericana Editores.

Hernández, V., Fernández, K. y Pulido, J. (2018). La actitud hacia la educación en línea en estudiantes universitarios. Revista de Investigación Educativa, 36(2), 349-364. https://doi.org/10.6018/rie.36.2.277451

Himmel, E. (2002). Modelo de análisis de la deserción estudiantil en la educación superior. Calidad en la Educación, (17), 91-108. https://doi. org/10.31619/caledu.n17.409

Lara, L. (2002). Análisis de los recursos interactivos en las aulas virtuales. [Ponencia]. Segundo Congreso Virtual «Integración sin barreras en el siglo

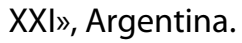

Liu, Á., Miranda, V. Quiroz, M. y Bazalar J. (2018). Identificar los factores que influyen en la deserción de los estudiantes en un instituto de educación superior tecnológica privada en la Ciudad de Lima. Universidad Tecnológica del Perú. 
Loreto, M. (2020). Educación como disciplina y como objeto de estudio: aportes para un debate. Desde el Sur, 12(1), 201-211. https://revistas.cientifica.edu.pe/index.php/desdeelsur/article/view/626

Montenegro, G. (2020). El desempeño docente y la práctica inclusiva en el nivel superior. Desde el Sur, 12(2), 437-452. https://revistas.cientifica.edu. pe/index.php/desdeelsur/article/view/671

Montoya, M. (1999). Extended stay at university: An application of multinomial logit and duration models. Applied Economics, 31(11), 1411-1422.

Morán, C. y Menezes, E. (2016). La motivación de logro como impulso creador de bienestar: su relación con los cinco grandes factores de la personalidad. INFAD, Revista de Psicología, 1(2), 31-40. http://dx.doi.org/10.17060/ ijodaep.2016.n1.v2.292

McKay, M., Davis, M. y Fanning, O. (1998). Técnicas cognitivas para el tratamiento del estrés. Martínez Roca.

Otzen, T. y Manterola, C. (2017). Técnicas de muestreo sobre una población a estudio. International Journal of Morphology, 35(1), 227-232. https:// dx.doi.org/10.4067/S0717-95022017000100037

Patiño, L. y Cardona, A. (2012). Revisión de algunos estudios sobre la deserción estudiantil universitaria en Colombia y Latinoamérica. Theoria, 21,(1), 9-20. https://www.redalyc.org/pdf/299/29931769002.pdf

Sistema Nacional de Evaluación, Acreditación y Certificación de la Calidad Educativa, Sineace (2020, 11 de diciembre). Encuentro virtual: Impacto de la situación excepcional causada por la pandemia COVID -19 en la calidad del servicio de la educación superior universitaria. [Archivo de video]. YouTube. https://youtu.be/-OWW1N-3qTQ

Spady, W. G. (1970). Dropouts from higher education: An interdisciplinary review and synthesis. Interchange, 1, 64-85.

Tinto, V. (1975). Dropouts from higher education: A theoretical synthesis of the recent literature. A Review of Educational Research, 45, 89-125.

Tinto, V. (1987). The principles of effective retention. ERIC, 1-18. https:// files.eric.ed.gov/fulltext/ED301267.pdf

Tinto, V. (1989). Definir la deserción: una cuestión de perspectiva. Revista de la Educación Superior, 33-51. http://publicaciones.anuies.mx/pdfs/revista/Revista71_S1A3ES.pdf

Unesco (2020). COVID-19 y educación superior: De los efectos inmediatos al día después. Análisis de impactos, respuestas políticas y recomendaciones. https://www.iesalc.unesco.org/wp-content/uploads/2020/04/ COVID-19-070420-ES-2-1.pdf 
Uribe, S. (2021). Educación superior en tiempos de Covid-19: Un análisis de los patrones de interrupción de estudios para el caso peruano. [Trabajo de suficiencia profesional para optar el título de economista, Universidad de Piura, Facultad de Ciencias Económicas y Empresariales].

Villarreal, Y., Castillo, S., Griffin, Y. y Rodríguez, K. (2011). Aproximación al estudio de los factores que inciden en el abandono de las asignaturas virtuales en la Universidad Tecnológica de Panamá. Congresos CLABES. https:// revistas.utp.ac.pa/index.php/clabes/article/view/1227

Recepción: 5/5/2021

Aceptación: 10/8/2021 